\title{
BMJ Open What's in a name? The challenge of describing interventions in systematic reviews: analysis of a random sample of reviews of non-pharmacological stroke interventions
}

\author{
Tammy C Hoffmann, ${ }^{1}$ Marion F Walker, ${ }^{2}$ Peter Langhorne, ${ }^{3}$ Sally Eames, ${ }^{1}$ \\ Emma Thomas, ${ }^{1}$ Paul Glasziou ${ }^{1}$
}

To cite: Hoffmann TC, Walker MF, Langhorne $P$, et al. What's in a name? The challenge of describing interventions in systematic reviews: analysis of a random sample of reviews of nonpharmacological stroke interventions. BMJ Open 2015;5:e009051.

doi:10.1136/bmjopen-2015009051

- Prepublication history for this paper is available online. To view these files please visit the journal online (http://dx.doi.org/10.1136/ bmjopen-2015-009051).

Received 14 June 2015 Accepted 16 October 2015

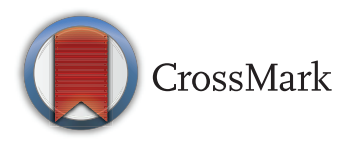

${ }^{1}$ Centre for Research in Evidence-Based Practice, Faculty of Health Sciences and Medicine, Bond University, Gold Coast, Queensland, Australia

${ }^{2}$ Faculty of Medicine and Health Sciences, University of Nottingham, Nottingham, UK

${ }^{3}$ Institute of Cardiovascular and Medical Sciences, University of Glasgow, Glasgow, UK

Correspondence to Professor Tammy C Hoffmann;

thoffmann@bond.edu.au

\section{ABSTRACT}

Objective: To assess, in a sample of systematic reviews of non-pharmacological interventions, the completeness of intervention reporting, identify the most frequently missing elements, and assess review authors' use of and beliefs about providing intervention information.

Design: Analysis of a random sample of systematic reviews of non-pharmacological stroke interventions; online survey of review authors.

Data sources and study selection: The Cochrane Library and PubMed were searched for potentially eligible systematic reviews and a random sample of these assessed for eligibility until 60 (30 Cochrane, 30 non-Cochrane) eligible reviews were identified.

Data collection: In each review, the completeness of the intervention description in each eligible trial ( $n=568)$ was assessed by 2 independent raters using the Template for Intervention Description and Replication (TIDieR) checklist. All review authors $(n=46)$ were invited to complete a survey.

Results: Most reviews were missing intervention information for the majority of items. The most incompletely described items were: modifications, fidelity, materials, procedure and tailoring (missing from all interventions in $97 \%, 90 \%, 88 \%, 83 \%$ and $83 \%$ of reviews, respectively). Items that scored better, but were still incomplete for the majority of reviews, were: 'when and how much' (in $31 \%$ of reviews, adequate for all trials; in $57 \%$ of reviews, adequate for some trials); intervention mode (in $22 \%$ of reviews, adequate for all trials; in $38 \%$, adequate for some trials); and location (in $19 \%$ of reviews, adequate for all trials). Of the $33(71 \%)$ authors who responded, $58 \%$ reported having further intervention information but not including it, and $70 \%$ tried to obtain information.

Conclusions: Most focus on intervention reporting has been directed at trials. Poor intervention reporting in stroke systematic reviews is prevalent, compounded by poor trial reporting. Without adequate intervention descriptions, the conduct, usability and interpretation of reviews are restricted and therefore, require action

\section{Strengths and limitations of this study}

- Analysis of intervention reporting in 568 trials from a random sample of 60 systematic reviews (both Cochrane and non-Cochrane reviews).

- Intervention descriptions in each eligible trial, in each review, were assessed by two independent raters using the Template for Intervention Description and Replication (TIDieR) checklist.

- A parallel survey of the corresponding author $(n=46)$ of each included review was also conducted.

- Response rate to the author survey was good $(71 \%)$, although there may have been recall bias and responses from authors who were interested in the topic.

- Our sample contained only reviews of nonpharmacological and non-surgical stroke interventions, limiting generalisability of the results.

by trialists, systematic reviewers, peer reviewers and editors.

\section{INTRODUCTION}

Inadequate descriptions of interventions in randomised trials, with crucial details missing from many, have been identified as a problem for both implementation of research and its synthesis. For nonpharmacological interventions, the problem is even greater, with more than half of trials lacking essential information about the interventions. ${ }^{1}$ A serious consequence of intervention details missing from trial reports is that it hampers clinicians from using the interventions in practice. Yet it is systematic reviews, rather than trials, that many clinicians turn to when looking for evidence. For effective interventions to make their way into practice, clinicians need to know the details 
of the intervention and be able to answer the question of 'How do I actually provide the intervention that this review is recommending?'

Exploration of the completeness of reporting of interventions in systematic reviews has received little attention. Among the recommendations of studies into the applicability and usability of systematic reviews, it has been noted that more detailed information about interventions should be included. ${ }^{2}{ }^{3}$ Lack of usefulness has been identified as a barrier to the uptake of evidence from systematic reviews. ${ }^{4}$ A preliminary analysis of 25 systematic reviews, sourced from a pool of reviews judged to be of high quality, found that only $12 \%$ of reviews had sufficient intervention information to be replicable (as assessed by a single question)..$^{5}$ Detailed analysis of the reporting of interventions in a sample that includes both Cochrane and non-Cochrane systematic reviews has not occurred.

While this issue is problematic for nearly all nonpharmacological interventions, some of the earliest work to identify effective components of complex nonpharmacological interventions and facilitate implementation of systematic review results was done for stroke. ${ }^{6}$ Rehabilitation is a major part of stroke care, ${ }^{7}$ yet evidence-practice gaps abound. Stroke rehabilitation is frequently acknowledged as a 'black box', ${ }^{8}$ with limited knowledge about what are the effective components of interventions and their features (eg, optimal intensity, duration and timing).

Uptake of evidence into practice is hampered by these knowledge gaps and a need for rehabilitation to be standardised based on the best evidence has been identified as one of the priorities of the stroke world agenda. ${ }^{9}$ However, progress towards evidence-based rehabilitation will be hindered if trials and reviews do not clearly describe their interventions. Using a sample of systematic reviews of non-pharmacological stroke interventions, this study aimed to: (1) assess the completeness of reporting of interventions in systematic reviews; (2) identify the most frequently missing elements in descriptions of interventions; and (3) survey review authors about their use of and beliefs about intervention information in systematic reviews.

\section{METHODS}

\section{Search strategy and selection of reports of trials}

To be eligible for inclusion, reviews had to be a systematic review of non-pharmacological and non-surgical interventions which were provided to a stroke population. Non-pharmacological interventions were defined as interventions, including rehabilitation, psychotherapy, behavioural interventions, non-surgical technical procedures and devices, and complementary medicine. ${ }^{1}$ Reviews which included trials of both nonpharmacological and pharmacological interventions were eligible for inclusion as a review, although only the non-pharmacological interventions were rated. Reviews were not eligible if they were reviews of only pharmacological or surgical interventions, or of diagnostic or screening tests.

We aimed to randomly select 30 Cochrane and 30 non-Cochrane systematic reviews of non-pharmacological and non-surgical stroke interventions. Though we did not do a formal sample size calculation, we were aware that 60 systematic reviews should have at least 500 studies (Cochrane reviews have a median of 8 trials; non-Cochrane, a median of 23) ${ }^{10}$ Hence, estimates of proportions would have $95 \%$ CIs better than $\pm 7 \%$ which we felt sufficient for the descriptive purposes of this study. We used a stratified sampling strategy, so that a matched number of Cochrane and non-Cochrane reviews were obtained. Our rationale for this is that Cochrane reviews are high profile and high impact reviews, but account for approximately $10 \%$ of all published reviews, ${ }^{11}$ and in a random sample of reviews, these would be under-represented. A medical librarian experienced in conducting systematic reviews helped develop and run the searches. We first searched the current (Issue 2, 2013) Cochrane Library for all completed reviews published by the Cochrane Stroke Group, which identified 156 Cochrane reviews on stroke. Two people (SE, TCH) independently screened titles and abstracts to identify reviews of non-pharmacological and non-surgical interventions $(n=58)$. We then developed a search strategy that included the MeSH terms used to index the selected Cochrane reviews and searched in PubMed with the strategy: MeSH descriptor[Stroke] explode all trees, with qualifiers of[Complications- CO, Psychology-PX, Rehabilitation-RH]; medline[tiab] OR (systematic[tiab] AND review[tiab]) OR meta-analysis[pt]; and excluding publications which had the Cochrane Database of Systematic Reviews as a source. The search was limited to the most recent 5 -year period (August 2008-2013) and produced 244 results.

The article identification numbers from all of the articles in both the Cochrane and non-Cochrane search results were separately exported to Excel. A computerbased random number generator was then used to select 30 reviews from each set of search results. The full text of the selected reviews was examined by two raters (SE, ET) to identify reviews that met the inclusion criteria as described above. This process continued until 30 reviews from each search (total of 60 reviews) were identified. Disagreements about unclear eligibility were resolved by discussion.

\section{Rating of intervention reporting in each systematic review}

For each included systematic review, the eligibility of each intervention in each trial was assessed before the intervention reporting was assessed. Interventions were eligible if they were: (1) a non-pharmacological intervention or a complex intervention in which the major focus was a non-pharmacological component; and (2) evaluated in a randomised trial. 
The completeness of reporting of each eligible intervention in each systematic review was assessed by two authors (SE, ET) independently using the Template for Intervention Description and Replication (TIDieR) guide and checklist, which was developed to provide authors and peer reviewers with guidance about the essential elements of interventions that should be reported. ${ }^{12}$ The TIDieR checklist contains 12 items (see table 1). For this study, item 1 (intervention name or phrase) was not scored as this is unlikely to be reported for each intervention in a systematic review. Item 12 (actual/extent of intervention fidelity) was scored as not applicable if item 11 (planning/assessment of intervention fidelity) was scored as inadequate. When trials contained evaluations of more than one eligible intervention (such as by using three groups or a factorial design), the reporting of each intervention was assessed separately. Items missing from the intervention description, or not described in sufficient detail for replication, were considered to be incomplete. For interventions which were assessed as being incompletely reported, we recorded comments about what was missing. If review authors reported that intervention details were described further in other sources (such as websites, online appendices or reference materials), we located and examined these additional sources before completing the rating of the checklist items. The raters completed the assessment of reviews in blocks of 5 and after each block assessment, discussed their ratings. Disagreements in ratings were resolved by discussion between the two assessors, with a third author (TCH) arbitrating where necessary.

\section{Survey of corresponding review authors}

For each systematic review, the corresponding author was emailed and invited to complete an online survey via SurveyMonkey. The survey contained eight questions (a mixture of open-ended, and fixed-choice questions with the option of providing additional comments) and asked authors: whether they had additional intervention information that was not reported in the review and the reason for this; whether they attempted to obtain additional intervention information and if so, methods used to obtain such information; details of additional information obtained; and the role of intervention descriptions in systematic reviews. If no response was received, a reminder email was sent after 2 weeks, and after a further 4 weeks.

\section{Data analysis}

Details of ratings and authors' survey responses were entered into Excel and used to analyse data descriptively. For each systematic review, we synthesised the ratings across the multiple interventions for each TIDieR item as: adequate for all (indicating that the item had been clearly reported for all of the eligible interventions in

Table 1 Brief description of the Template for Intervention Description and Replication (TIDieR) items that were used to assess intervention reporting

\begin{tabular}{|c|c|c|}
\hline Item number* & Item name & Item description \\
\hline 2 & Why & Describe any rationale, theory or goal of the elements essential to the intervention \\
\hline 3 & What: materials & $\begin{array}{l}\text { Describe any physical or informational materials used in the intervention, including those } \\
\text { provided to participants or used in intervention delivery or in training of intervention } \\
\text { providers; provide information on where the materials can be accessed (eg, online } \\
\text { appendix, URL) }\end{array}$ \\
\hline 4 & What: procedures & $\begin{array}{l}\text { Describe each of the procedures, activities, and/or processes used in the intervention, } \\
\text { including any enabling or support activities }\end{array}$ \\
\hline 5 & Who provided & $\begin{array}{l}\text { For each category of intervention provider (eg, psychologist, nursing assistant), describe } \\
\text { their expertise, background and any specific training given }\end{array}$ \\
\hline 6 & How & $\begin{array}{l}\text { Describe the modes of delivery (eg, face-to-face or by some other mechanism, such as } \\
\text { internet or telephone) of the intervention and whether it was provided individually or in a } \\
\text { group }\end{array}$ \\
\hline 7 & Where & $\begin{array}{l}\text { Describe the type(s) of location(s) where the intervention occurred, including any } \\
\text { necessary infrastructure or relevant features }\end{array}$ \\
\hline 8 & $\begin{array}{l}\text { When and how } \\
\text { much }\end{array}$ & $\begin{array}{l}\text { Describe the number of times the intervention was delivered and over what period of } \\
\text { time, including the number of sessions, their schedule, and their duration, intensity or } \\
\text { dose }\end{array}$ \\
\hline 9 & Tailoring & $\begin{array}{l}\text { If the intervention was planned to be personalised, titrated or adapted, then describe } \\
\text { what, why, when and how }\end{array}$ \\
\hline 10 & Modifications & $\begin{array}{l}\text { If the intervention was modified during the course of the study, describe the changes } \\
\text { (what, why, when and how) }\end{array}$ \\
\hline 11 & How well: planned & $\begin{array}{l}\text { If intervention adherence or fidelity was assessed, describe how and by whom, and if } \\
\text { any strategies were used to maintain or improve fidelity, describe them }\end{array}$ \\
\hline $12^{*}$ & How well: actual & $\begin{array}{l}\text { If intervention adherence or fidelity was assessed, describe the extent to which the } \\
\text { intervention was delivered as planned }\end{array}$ \\
\hline
\end{tabular}

*Item 1 was not scored for this study; item 12 was scored as 'not applicable' if item 11 was scored as inadequate. 
the review); adequate for some (indicating that the item had been described clearly for some, but not all, of the eligible interventions in the review); or adequate for none (indicating that the item had been not described clearly or not at all for any of the interventions in the review). The broad interventions covered by the eligible reviews (as shown in table 2) were categorised by the two raters (ET, SE) according to the National Stroke Foundation's Stroke Guidelines categories. ${ }^{13}$ When comparing Cochrane and non-Cochrane reviews, we collapsed the 'adequate for all' and 'adequate for some' into one category. Authors' responses to open-ended survey responses were independently coded into thematic clusters by two authors (TCH, SE), with any discrepancies discussed until consensus was reached.

\section{RESULTS}

Our sample of 60 systematic reviews included a total of 568 eligible trials and 589 eligible interventions (mean of 10.1 eligible interventions per Cochrane review, range $0-51$; mean of 9.5 eligible interventions per non-Cochrane review, range 6-28). Table 2 shows the broad interventions covered by the reviews, along with the number of eligible interventions. Two Cochrane reviews identified no studies eligible for inclusion and were not able to be analysed further for this study.

\section{Completeness of intervention descriptions in systematic reviews}

Most reviews were missing information for the majority of items (figure 1). The most incompletely described items were: modifications, intervention fidelity, materials, procedure and tailoring (missing from all interventions in $97 \%, 90 \%, 88 \%, 83 \%$ and $83 \%$ of reviews, respectively). Items that scored higher, but still incomplete for the majority of reviews, were: details of 'when and how much' of the intervention (in $31 \%$ of reviews, it was adequate for all interventions; in $57 \%$ of reviews, it was adequate for some interventions); how (ie, the mode) the intervention was provided (in 22\% of reviews, it was adequately reported for all interventions; in 38\% of reviews, it was adequately reported for some interventions); and where (setting) the intervention was provided (in 19\% of reviews, it was adequate for all interventions; and adequate for some interventions in $19 \%$ of reviews). Only two reviews, both non-Cochrane reviews, directed readers to other sources for additional intervention information. In both of these reviews, supplemental tables contained additional data, and as part of this, also contained information about item 8 ('when and how much' of the intervention).

The completeness of intervention reporting of Cochrane reviews compared with non-Cochrane reviews is shown in figure 2, where, for each TIDieR item, the mean percentage of systematic reviews in which there were adequate description of all or some of the interventions in the review is presented. Reporting of details of interventions was clearly better (in $\geq 20 \%$ of reviews) in
Cochrane reviews for 5 of the 11 items: intervention rationale; procedure; intervention provider; how the intervention was delivered; and where the intervention was delivered. For item 11 (assessment of intervention fidelity or adherence), there was a slightly higher percentage of non-Cochrane reviews (13\%), compared with Cochrane reviews $(7 \%)$, in which some or all of the interventions in the reviews described this item, although this item was only reported in a very small proportion of reviews. For the remaining five items (materials; when and how much; tailoring; modifications; actual/extent of fidelity), there was little difference between the completeness of reporting in Cochrane reviews compared with non-Cochrane reviews.

\section{Survey of review corresponding authors}

We contacted the 46 corresponding authors of the 58 reviews with eligible interventions (several authors were corresponding authors for two or more reviews). Of the $33(71 \%)$ authors who responded, $19(58 \%)$ reported that they had further information about the intervention that was not included in the review, while $23(70 \%)$ indicated that they had tried to obtain further information about the intervention as part of the review, using various methods, with variable success. Reasons for not attempting to obtain further intervention information included: not perceived as necessary as part of a review, not needed because interventions were already fully described, or would not subsequently have been able to incorporate the information into the review.

A summary of responses to questions where authors could provide comments and elaborate on these issues is shown in table 3. Some authors acknowledged the importance of completely describing interventions in reviews, with multiple reasons given, such as helping with decisions about study inclusion, appropriateness of data synthesis and subgroup analysis, and facilitating the use of interventions in practice. Some authors commented that reviews typically do not provide sufficient intervention information, while others commented that such information could be useful as part of the review process, but did not need to be included in the review itself. Suggested contributors to the problem were lack of details provided in the original trials and word limits (for both trials and reviews).

\section{DISCUSSION}

We found a high rate of incomplete reporting of intervention items in systematic reviews, with details describing most intervention elements missing from the majority of the reviews. This has important consequences for the conduct, interpretation and use of the reviews. As trials are synthesised into reviews, the problem of intervention details missing from trial publications is compounded. Lack of intervention detail affects more than the usability and interpretation of a completed review. It can also hamper steps in the review 
Table 2 Categories of interventions of the included systematic reviews, the number of eligible studies and interventions, and excluded studies

\begin{tabular}{|c|c|c|c|c|c|}
\hline \multirow[b]{2}{*}{ Intervention category } & \multicolumn{2}{|c|}{ Systematic reviews } & \multicolumn{2}{|c|}{$\begin{array}{l}\text { Number of eligible studies } \\
\text { (interventions) included }\end{array}$} & \multirow[b]{2}{*}{$\begin{array}{l}\text { Number of excluded } \\
\text { studies (main reason) }\end{array}$} \\
\hline & $\begin{array}{l}\text { Cochrane } \\
\text { reviews }(n)\end{array}$ & $\begin{array}{l}\text { Non-Cochrane } \\
\text { reviews }(n)\end{array}$ & $\begin{array}{l}\text { Cochrane } \\
\text { reviews }\end{array}$ & $\begin{array}{l}\text { Non-Cochrane } \\
\text { reviews }\end{array}$ & \\
\hline \multicolumn{6}{|l|}{ Organisation of service } \\
\hline $\begin{array}{l}\text { Care after hospital } \\
\text { discharge }\end{array}$ & 5 & 1 & $26(26)$ & $15(12)$ & \\
\hline Hospital care & 2 & 1 & $50(50)$ & $4(4)$ & 5 (not RCTs) \\
\hline $\begin{array}{l}\text { Patient and carer/family } \\
\text { support }\end{array}$ & 1 & 0 & $16(18)$ & $0(0)$ & \\
\hline \multicolumn{6}{|l|}{ Rehabilitation } \\
\hline Upper limb & 2 & 11 & $24(24)$ & $106(114)$ & $\begin{array}{l}10 \text { (not stroke } \\
\text { population); } \\
17 \text { (not RCTs) }\end{array}$ \\
\hline Physical activity & 2 & 5 & $13(13)$ & $50(51)$ & 13 (not RCTs) \\
\hline $\begin{array}{l}\text { Amount, intensity and } \\
\text { timing }\end{array}$ & 1 & 0 & $1(1)$ & $0(0)$ & \\
\hline Cognition & 5 & 2 & $23(23)$ & $7(9)$ & 7 (not RCTs) \\
\hline Communication & 1 & 0 & $39(51)$ & $0(0)$ & \\
\hline $\begin{array}{l}\text { Sensorimotor } \\
\text { impairment }\end{array}$ & 2 & 4 & $13(13)$ & $51(48)$ & 7 (not stroke population) \\
\hline Activities of daily living & 2 & 0 & $18(21)$ & $0(0)$ & \\
\hline \multicolumn{6}{|l|}{ Complications } \\
\hline Mood disturbance & 1 & 1 & 2 (2) & $6(5)$ & 4 (pharmacological) \\
\hline $\begin{array}{l}\text { Loss of cardiorespiratory } \\
\text { fitness }\end{array}$ & 1 & 2 & $32(32)$ & $17(15)$ & 1 (not RCT) \\
\hline Subluxation & 2 & 0 & $18(19)$ & $0(0)$ & \\
\hline Poor hygiene & 1 & 0 & $3(3)$ & $0(0)$ & \\
\hline Fatigue & 1 & 0 & $1(1)$ & $0(0)$ & 2 (pharmacological) \\
\hline $\begin{array}{l}\text { Complementary and } \\
\text { alternative therapy for } \\
\text { acute management }\end{array}$ & 1 & 1 & $7(7)$ & $8(8)$ & \\
\hline $\begin{array}{l}\text { Lifestyle modification for } \\
\text { secondary prevention }\end{array}$ & 0 & 2 & $0(0)$ & $18(19)$ & $\begin{array}{l}3 \text { (pharmacological); } 17 \\
\text { (protocols) }\end{array}$ \\
\hline Total & \multicolumn{2}{|c|}{$\begin{array}{l}\text { Cochrane reviews } \\
\text { Non-Cochrane reviews }\end{array}$} & \multicolumn{2}{|c|}{$\begin{array}{l}286 \text { studies ( } 304 \text { interventions) } \\
282 \text { studies ( } 285 \text { interventions) }\end{array}$} & \\
\hline
\end{tabular}

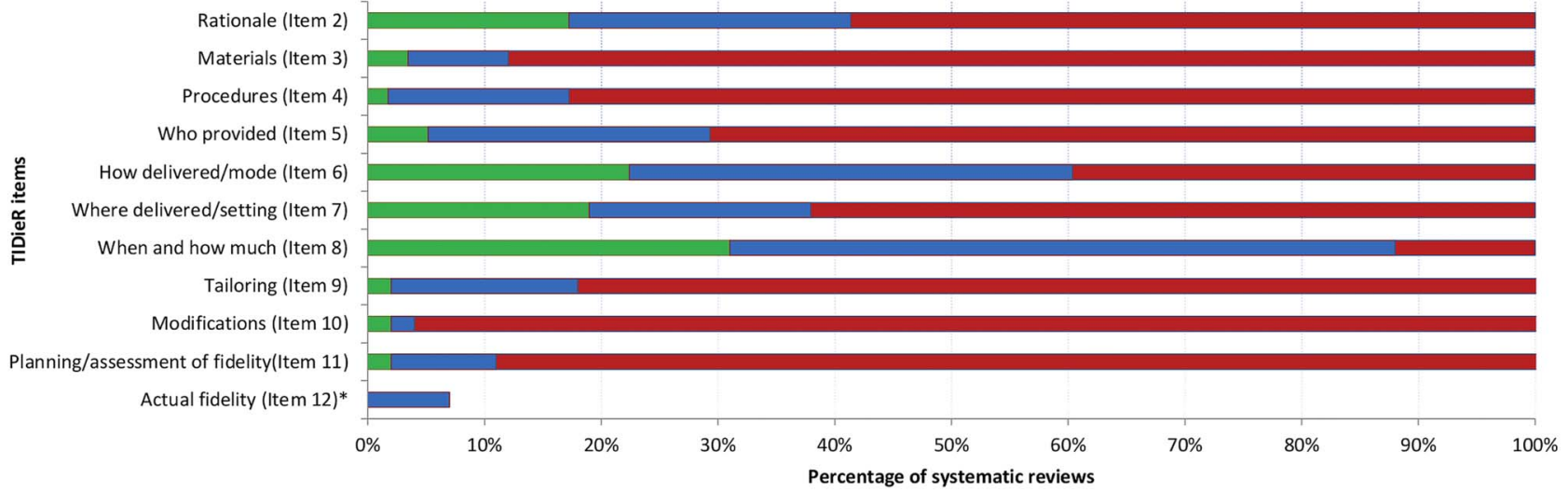

all interventions adequately reported in the review $\square$ Some interventions adequately reported in the review $\quad$ No interventions adequately reported in the review

* rated as N/A if Item 11 rated as 'no'

Figure 1 Percentage of systematic reviews, per checklist item, in which intervention descriptions were 'adequate for all interventions', 'adequate for some' and 'adequate for none' of the interventions included in each review. 


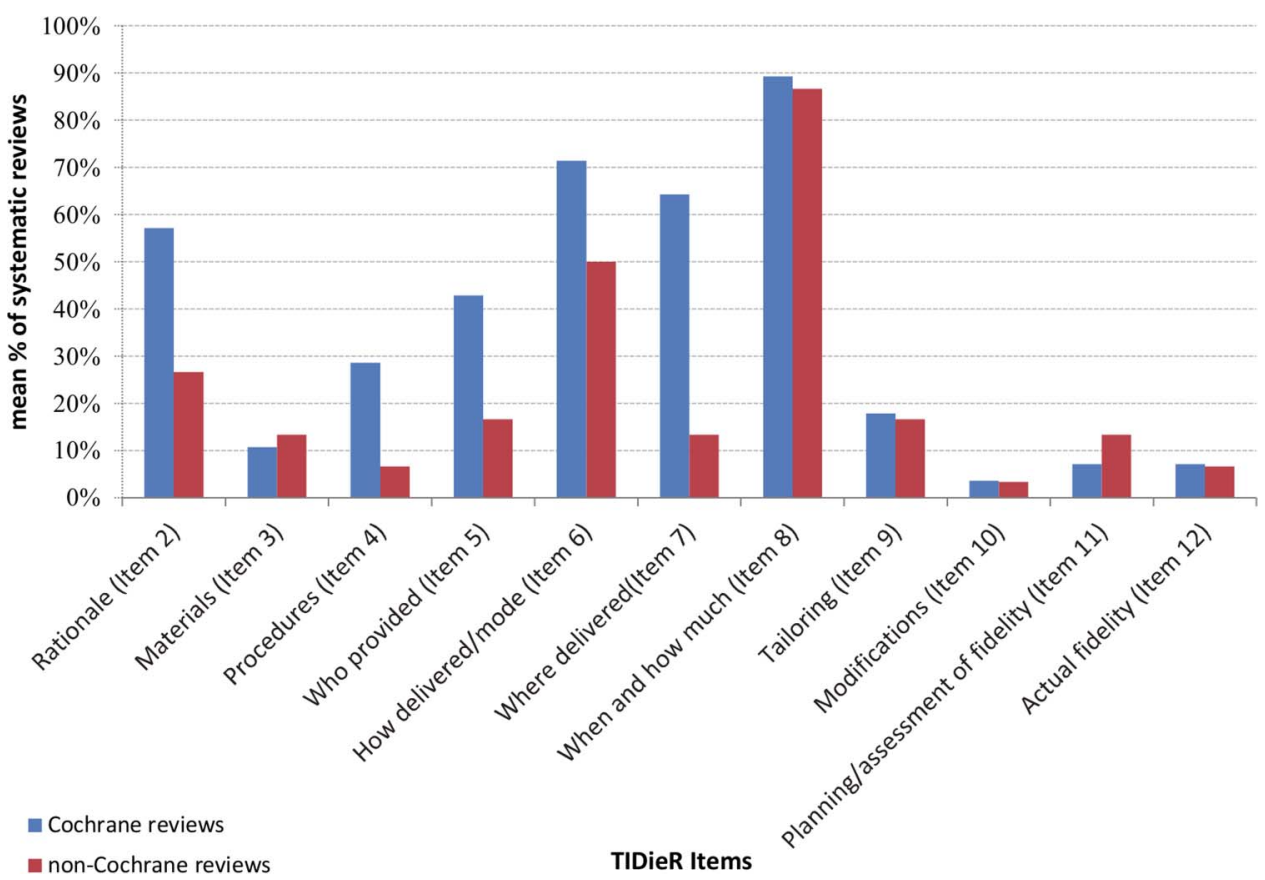

process, such as decisions about eligibility, meta-analysis and subgroup analysis. Some review authors were conscious of the problem and attempted to obtain additional information, with variable success.

Strengths of this study include: the use of a comprehensive checklist and two independent raters to assess intervention reporting; the number and variety of reviews included studies and interventions; inclusion of both Cochrane and non-Cochrane reviews; and a good response rate to our author survey. Some of the limitations of the study are that our sample of reviews only contains non-pharmacological and non-surgical interventions, and only stroke interventions and hence, the Cochrane reviews are from only one Cochrane review group. The extent to which our random sample reflects systematic reviews in stroke and the generalisability of these results to other conditions and interventions is unknown. Although the response rate to our author survey was good $(71 \%)$, authors with an interest in the issue may have been more likely to respond, whereas authors of older reviews may not have responded or been able to recall information about the questions asked, and a small number of the authors had authored more than one of the included reviews. We did not formally measure a single level of agreement between the raters primarily because an iterative process was used with the raters comparing ratings after every five reviews. There were no items which raters consistently disagreed on or were notably more problematic than others.

Analysis of the reporting of the intervention elements in systematic reviews has rarely been studied. Assessment of the intervention reporting in 25 Cochrane reviews of acupuncture found that many reviews lacked intervention details, even though the details were often reported in the trials included in the reviews, ${ }^{14}$ reinforcing that lack of details in trials is only one contributor to the problem. In this study, intervention elements that were mostly absent from nearly all reviews include details of intervention procedures, materials, tailoring, modifications and fidelity. These are the elements that may be perceived as the most difficult to describe and require the most words. Yet, for intervention replication, they are among the most crucial. Items 3 (materials) and 4 (procedures) have been described as analogous to the ingredients and methods of a recipe ${ }^{12}$-without these details, the intervention cannot be provided with fidelity. These elements have also been reported as the elements most frequently missing from publications of randomised trials of non-pharmacological interventions; ${ }^{1}$ thus, it is not surprising that these are also among the most frequently missing elements in reviews. A particular challenge with these elements is the amount of detail required to report them adequately. Elements that are easier and more succinct to report, such as the "when and how much' of the intervention and its mode of delivery, were better reported. In Cochrane reviews, these and other intervention details were typically found in the Characteristics of Included Studies tables, rather than in the main text of the review.

For almost half of the TIDieR items, Cochrane reviews more completely reported intervention details than non-Cochrane reviews and for all but one of the remaining items, there was no difference between the two samples of reviews. Better quality reporting of Cochrane 
Table 3 Survey questions to and responses from corresponding authors $(\mathrm{n}=33)$

Q. Briefly describe why you did not include this information in the review. Category of response (number of authors providing a response in each category) and illustrative quotes

Was not relevant (2)

"Didn't think it was relevant"

Page/word limits (3)

- "No detailed description of the interventions because of word limit and because of clarity. Was out of the focus of the review."

Not included/reported in the review, but used in the review process (6)

- "Some of this information was incorporated... when the meta-analyses were being conducted. Without including this information, the study might not have met inclusion and therefore, would have been excluded."

"Some data contributed to meta-analysis of outcome data"

- "Only used to determine eligibility for inclusion in review"

" "Intervention details were not used much in the review but were mostly used to make decisions about inclusion, or sub group allocation"

Other (2)

- "Was raised... as points of difference in the discussion."

"Other reviews covered the interventions...but not with a stroke- specific population."

Q. If you tried to obtain further information about the intervention/s, how and where from? ( $n=23)$ (could choose $\geq 1$ response)

Method of obtaining further information

Email/fax

Searching for other publications related to original trial/s

Searching for published protocol of original trial/s

Searching clinical trial registries

Face-to-face (eg, semistructured interviews with trialists at conferences)

Telephone

Other (broad intervention approach described in a book, but not specifically about the trial)

$Q$. If you contacted authors of the trial/s for further intervention information, did the trial author/s respond? ( $n=22$ )(could choose $\geq 1$ response)

Response

All authors who were contacted responded, and provided further information

Some authors who were contacted responded, and provided further information

Some authors responded, but did/could not provide further information (eg, data were so old they were no longer available)

None of the authors who were contacted provided further information

None of the authors responded

Q. If trial authors provided you with additional intervention information, please briefly describe the nature/format of this information. Category of response

(number of authors providing a response) and illustrative quotes Details about intervention components (9)

- "Explained what was meant by intervention terms or described tools used for intervention or amount, etc"

- "Greater detail about components of the intervention, such as intensity, duration, application details, instructions, etc"

Intervention protocols and intervention materials (3)

- "Intervention protocols, greater details about intervention type, implementation, intensity, staff training outlines, descriptions..."

"Authors sent information/material resources used in the intervention, provided a website with more information, provided greater detail about a component of the intervention, such as intensity or duration"

- "Internal reports, draft papers, local protocols"

Q. If you did not attempt to obtain further information about the intervention/s, was there a reason? (provide main reason only) $(n=18)$

Response from author

Not needed as interventions were fully described in the original trial 


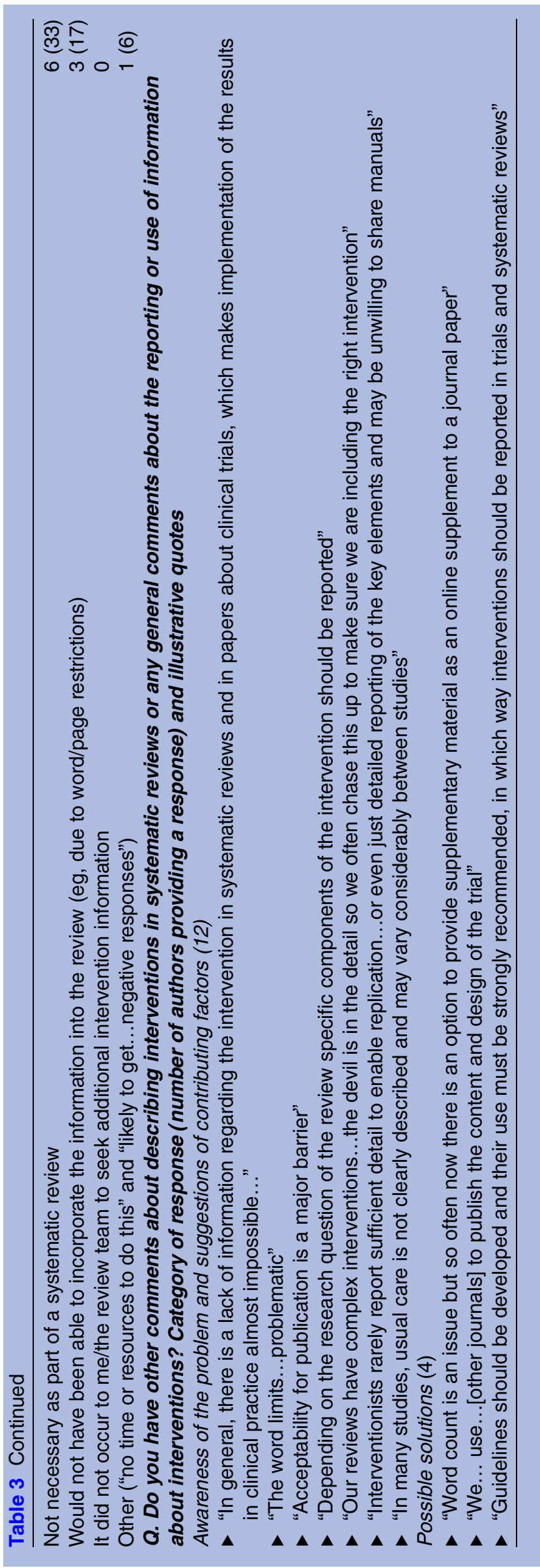

reviews than non-Cochrane reviews has been previously identified in a cross-sectional sample of reviews ${ }^{10}$ and a sample of stroke rehabilitation systematic reviews, ${ }^{15}$ although these studies did not examine intervention reporting. Among the reasons suggested for better reporting in Cochrane reviews are the absence of word limits, and detailed guidance and policies on review conduct and reporting. ${ }^{10}$ A number of the authors surveyed mentioned word limits as a barrier to reporting intervention details, although presumably these were authors of non-Cochrane reviews. For reviews where word limits may prevent detailed reporting, authors are encouraged to provide intervention details in online supplementary materials, which is now an option in the majority of journals. ${ }^{16}$

However, having no word limit on reviews is clearly not sufficient to ensure complete reporting of intervention details. As lack of awareness among authors of trials about what constitutes an adequate description of an intervention contributes to poor reporting of trials, ${ }^{17}$ it may also be problematic for review authors as guidance for review authors about how to report interventions is lacking. The most relevant section (7.3.4) of the current version (V.5.1.0) of the Cochrane Handbook (handbook.cochrane.org) does mention that reviews should provide information that is 'sufficient for replication', and gives a few examples of what this might include (eg, route of delivery, dose, format, timing). However, this guidance does not appear in the most recent version (V.2.3) of Cochrane's 'Methodological standards for the conduct of Cochrane Intervention Reviews', the most relevant mandatory standard (C44) simply advises that "basic characteristics of each study will need to be presented..., including details of participants, interventions and comparators...". ${ }^{18}$ Other standards that are relevant but unlikely to be sufficient guidance to facilitate adequate intervention reporting include: a nonmandatory standard (C49) that encourages review authors to contact study authors for details of unpublished information, including 'details of interventions'; and a mandatory standard (C7) that requires any restrictions (ie, those that are ineligible) on interventions and comparators to be predefined and explained. While these standards continue to undergo refinement, the 2012 version (V.1.1) of these standards provided more details about how interventions should be reported and encouraged authors to report 'in a way that allows the intervention to be replicated', ${ }^{19}$ whereas this has been removed in the 2013 version. ${ }^{18}$

For non-Cochrane reviews, authors may use the PRISMA (Preferred Reporting Items for Systematic Reviews and Meta-analyses) statement as reporting guidance $;^{20}$ yet it also does not provide guidance about how to report interventions and only requests 'study characteristics'. Review authors may find the newly developed statement that guides intervention reporting for any evaluative study design (TIDieR) useful when considering what intervention elements they need details 
about while conducting, and particularly when writing, the review. Another tool which may help during the review process is the Oxford Implementation Index ${ }^{21}$ which aims to assist reviewers to extract and compare implementation data (includes intervention design) across trials.

Poor reporting of intervention details in systematic reviews is part of the broader problem of how to implement the results of systematic reviews. A recent paper ${ }^{22}$ described three possible approaches to selecting and implementing a specific intervention from a systematic review. Two of these approaches are dependent on sufficient intervention details being able to be obtained and the other, the simplest approach (single-trial-based choice), requires at least some interventions in the review to be replicable and for agreement about what constitutes the 'best' intervention. One way that review authors can help review users to consider which interventions might be most appropriate for their circumstance involves providing an 'intervention options table'. ${ }^{22}$ This table could summarise the pros and cons of the usable and feasible interventions in the review (and the criteria used to decide this), including elements of each version of the intervention studied and differences between trials' interventions.

In many reviews, authors commented (typically in the Discussion) that lack of intervention detail made synthesising, interpreting and using the results difficult or that trialists should clearly define each intervention tested. Reflecting this, some authors surveyed reported contacting trial authors for additional intervention information-some with success. Those that were able to obtain additional details described using the information in various parts of the review process, such as deciding on study eligibility and the appropriateness and interpretation of subgroup and meta-analyses. While some review authors viewed intervention details as important in both the conduct and reporting of the review, others did not include detailed intervention information in the review report or think it was necessary. Although many systematic review users are aware of the need to critically appraise them before deciding whether to use them, appraisal tools typically do not consider the adequacy of intervention reporting and how that may have influenced the review's steps and results. Future research that explores whether the completeness of intervention reporting (of both the experimental and comparison interventions) is related to variables, such as the quality of the review, would be valuable.

Most of the focus of poor intervention reporting as a barrier to evidence interpretation and uptake has been directed at the primary trials. This study highlights an even greater problem in systematic reviews, where the poor reporting of interventions in trials is compounded. This reinforces the importance of trialists providing sufficient intervention details. This issue is not unique to intervention details; a recent analysis of selective reporting bias of harms outcomes identified that harms data are incompletely reported in many primary studies and also in subsequent systematic reviews. ${ }^{23}$ Both trialists and systematic review authors have an important role to play in ensuring that interventions are adequately reported in systematic reviews. Poor reporting in the trials, however, is not the sole contributor to the inadequacies of intervention reporting in systematic reviews. As neither the current PRISMA statement or the Cochrane methodological standards provide guidance about intervention reporting, authors of reviews should consider also using guidance about intervention reporting and/or a structured method of reporting intervention details, and peer reviewers and editors should be cognisant of the adequacy with which interventions are reported in reviews. Having systematic reviews which contain adequate intervention information will assist at multiple stages of the review process as well as the interpretation of review results. Ultimately, this will help clinicians to implement effective interventions into practice.

Twitter Follow Tammy Hoffmann at @Tammy_Hoffmann

Acknowledgements The authors thank the authors of the reviews who responded to the survey and Sarah Thorning (Medical Librarian, Centre for Research in Evidence-Based Practice, Bond University) for her assistance with the searches

Contributors TCH was primarily responsible for study conception, design, and data analysis and interpretation. All authors had full access to all of the data in the study, and take responsibility for the integrity of the data and the accuracy of the data analysis. SE and ET rated the intervention descriptions. $\mathrm{TCH}$ led the writing of the first draft of the manuscript, and all authors contributed to drafting and revising the manuscript.

Funding TCH is supported by a National Health and Medical Research Council of Australia (NHMRC)/Primary Health Care Research Evaluation and Development Career Development Fellowship (number: 1033038) with funding provided by the Australian Department of Health and Ageing. PG is supported by a NHMRC Australia Fellowship (number: 527500).

Competing interests TCH and PG are part of the author team that developed the TIDieR statement. PL is a co-author on some of the included reviews and is Coordinating Editor of the Cochrane Stroke group, and $\mathrm{TCH}$ is a member of its Editorial Board.

Provenance and peer review Not commissioned; externally peer reviewed.

Data sharing statement No additional data are available.

Open Access This is an Open Access article distributed in accordance with the Creative Commons Attribution Non Commercial (CC BY-NC 4.0) license, which permits others to distribute, remix, adapt, build upon this work noncommercially, and license their derivative works on different terms, provided the original work is properly cited and the use is non-commercial. See: http:// creativecommons.org/licenses/by-nc/4.0/

\section{REFERENCES}

1. Hoffmann TC, Erueti C, Glasziou PP. Poor description of non-pharmacological interventions: analysis of consecutive sample of randomised trials. BMJ 2013;347:f3755.

2. Glenton $\mathrm{C}$, Underland $\mathrm{V}, \mathrm{Kho} \mathrm{M}$, et al. Summaries of findings, descriptions of interventions, and information about adverse effects would make reviews more informative. J Clin Epidemiol 2006;59:770-8.

3. Burford B, Lewin S, Welch V, et al. Assessing the applicability of findings in systematic reviews of complex interventions can enhance the utility of reviews for decision making. J Clin Epidemiol 2013;66:1251-61. 
4. Wallace J, Nwosu B, Clarke M. Barriers to the uptake of evidence from systematic reviews and meta-analyses: a systematic review of decision makers' perceptions. BMJ Open 2012;2:e001220.

5. Glasziou $P$, Meats $E$, Heneghan $C$, et al. What is missing from descriptions of treatment in trials and reviews? BMJ 2008;336:1472-4.

6. Langhorne P, Pollock A. What are the components of effective stroke unit care? Age Ageing 2002;31:365-71.

7. Langhorne P, Bernhardt J, Kwakkel G. Stroke rehabilitation. Lancet 2011;377:1693-702.

8. DeJong G, Horn SD, Conroy B, et al. Opening the black box of post-stroke rehabilitation: stroke rehabilitation patients, processes, and outcomes. Arch Phys Med Rehabil 2005;86:S1-7.

9. Hachinski V, Donnan GA, Gorelick PB, et al. Stroke: working toward a prioritized world agenda. Stroke 2010;41:1084-99.

10. Moher D, Tetzlaff J, Tricco AC, et al. Epidemiology and reporting characteristics of systematic reviews. PLoS Med 2007; 4:e78.

11. Bastian H, Glasziou P, Chalmers I. Seventy-five trials and eleven systematic reviews a day: how will we ever keep up? PLoS Med 2010;7:e1000326.

12. Hoffmann TC, Glasziou PP, Boutron I, et al. Better reporting of interventions: template for intervention description and replication (TIDieR) checklist and guide. BMJ 2014;348:g1687.

13. National Stroke Foundation. Clinical guidelines for stroke management 2010. Melbourne, Australia: National Stroke Foundation, 2010.

14. Kim KH, Kang JW, Lee MS, et al. Assessment of the quality of reporting for treatment components in Cochrane reviews of acupuncture. BMJ Open 2014;4:e004136.
15. Santaguida $\mathrm{P}$, Oremus $\mathrm{M}$, Walker $\mathrm{K}$, et al. Systematic reviews identify important methodological flaws in stroke rehabilitation therapy primary studies: review of reviews. J Clin Epidemiol 2012:65:358-67.

16. Hoffmann TC, English T, Glasziou Paul P. Reporting of interventions in randomised trials: an audit of journal Instructions to authors. Trials 2014;15:20.

17. Schroter S, Glasziou P, Heneghan C. Quality of descriptions of treatments: a review of published randomised controlled trials. BMJ Open 2012;2:e001978.

18. Chandler J, Churchill R, Higgins J, et al. Methodological standards for the conduct of new Cochrane Intervention Reviews. London, UK: Cochrane Collaboration, 2013.

19. Cochrane Editorial Unit. Methodological expectations of Cochrane Intervention Reviews. Standards for the reporting of new Cochrane Intervention Reviews. London, UK, 2012.

20. Moher D, Liberati A, Tetzlaff $J$, et al. Preferred reporting items for systematic reviews and meta-analyses: the PRISMA statement PLOS Med 2009;6:e1000097.

21. Montgomery P, Underhill K, Gardner F, et al. The Oxford Implementation Index: a new tool for incorporating implementation data into systematic reviews and meta-analyses. J Clin Epidemiol 2013:66:874-82.

22. Glasziou P, Chalmers I, Green S, et al. Intervention synthesis: a missing link between a systematic review and practical treatment(s). PLoS Med 2014:11:e1001690.

23. Saini P, Loke YK, Gamble C, et al. Selective reporting bias of harm outcomes within studies: findings from a cohort of systematic reviews. BMJ 2014;349:g6501. 